\title{
Laser And Nonlinear Optical Materials For Laser Remote Sensing
}

\author{
Norman P. Barnes \\ NASA Langley Research Center \\ Hampton, VA 23681
}

\section{Abstract}

NASA remote sensing missions involving laser systems and their economic impact are outlined. Potential remote sensing missions include: green house gasses, tropospheric winds, ozone, water vapor, and ice cap thickness. Systems to perform these measurements use lanthanide series lasers and nonlinear devices including second harmonic generators and parametric oscillators. Demands these missions place on the laser and nonlinear optical materials are discussed from a materials point of view. Methods of designing new laser and nonlinear optical materials to meet these demands are presented.

\section{Introduction:}

Measurements on the state of health of Planet Earth are required to serve as the basis for rational policy decisions. Among the measurements needed are: green house gasses, ozone, tropospheric winds, and ice cap thickness. Some possible policy decisions, such as limiting green house gas emission, can have huge economic impacts. The necessary measurements can be made on a global basis employing lidars deployed in polar orbiting satellites. The laser transmitters for these lidar systems are usually highly specialized. As an example the wavelength of the laser must coincide with the peak absorption of the atmospheric constituent of interest to within a part in $10^{6}$. Engineering and selection of the best laser and nonlinear optical materials for these applications can be facilitated by compositional tuning.

\section{Solid state laser materials:}

Solid state laser materials can be compositionally tuned to achieve the requisite wavelengths to remotely sense atmospheric variables such as water vapor and green house gasses. For space deployment, lanthanide series lasers are usually preferred over transition metal lasers. Lanthanide series lasers involve $4 f$ electronic transitions. Because the $4 \mathrm{f}$ electrons are shielded from the electric field of the crystal lattice by the $5 \mathrm{~s}$ and $5 p$ electrons, the transitions are usually narrow. On the other hand, transition metal lasers involve $3 d$ electronic transitions. These electrons are not shielded from the electric field of the 
crystal lattice and the transitions are usally quite wide. The product of the emission cross section, lifetime, and bandwidth is limited. Wide transitions of the transition metal lasers imply small emission cross sections, short lifetimes or both. The latter qualities are incompatible with diode pumping and efficient operation. Conversely, lanthanide series lasers are compatible with efficient diode pumping. However, the narrow band of wavelengths associated with a laser transition between 2 energy levels must nearly match the absorption feature of the atmospheric constituent of interest. Compositional tuning is a method of achieving this.

A review of the physics of lanthanide series lasers points out the degree of tuning possible. Energy levels of the $4 \mathrm{f}$ electrons depend both on the particular lanthanide series atom and the laser material in which it is found. Quantum mechanical effects affecting the $4 f$ electrons are: the central potential, the mutual repulsion of other electrons, the spin orbit effect, and the Stark splitting caused by the crystal field of the laser material. The first 3 effects are a function of the particular lanthanide series atom. On the other hand, Stark splitting is dependent on the local electric field caused by the particular laser material. Stark splitting lifts much of the degeneracy and spreads the energy levels associated with a particular manifold over a few hundred $\mathrm{cm}^{-1}$. As an example, the strong $\mathrm{Nd}$ transition in Nd:YAG is $1.064 \mu \mathrm{m}$ while the strong $\mathrm{Nd}$ transition in Nd:YLF is $1.047 \mu \mathrm{m}$. Suppose a wavelength between them is sought. Compositional tuning may be employed toward this end.

Splitting of energy levels within a manifold is described by the crystal field parameters, $B_{n m}$. Given the crystal field parameters, quantum mechanics can be used to predict the energy levels and thus the wavelengths. In turn, the crystal field parameters are a product of parameters that depends only on the particular lanthanide series atom, $\rho_{\mathrm{n}}$, and parameters that depend only on the crystal field, $A_{n m}$. Thus,

$$
B_{n m}=\rho_{n} A_{n m} .
$$

In practice, the $\rho_{n}$ parameters can be determined by fitting a set of experimental energy levels for a particular lanthanide series atom in a particular laser material and using calculated values for $A_{n m}$. Once this is done for any particular laser material, the derived $\rho_{n}$ parameters can be used for other laser materials. In practice, this may be done utilizing the experimental energy levels for several laser materials and averaging the results. 
$A_{n m}$ parameters can be calculated given the crystal lattice parameters and effective charge on the various atoms comprising the crystal lattice. Lattice parameters were measured for many crystals from X-ray data. Effective charge is often set as if the laser material were completely ionic. While this works for some laser materials better results are achieved using Pauling's theory of electro negativity to set effective charge. Crystal field components, $A_{n m}$, calculated utilizing this approach were successfully employed to invent a novel laser at a preselected wavelength useful for the remote sensing of water vapor. The wavelength of almost every laser material is experimentally measured when the laser material is demonstrated. Here a laser material that produces a preselected wavelength is theoretically predicted and then experimentally confirmed.

A laser material that produces an output at $0.9441 \mu \mathrm{m}$ was predicted utilizing compositional tuning and experimentally confirmed. $N d: Y A G$, that is $N d: Y_{3} A_{1} \mathrm{O}_{12}$, operates on the $R_{1}$ to $Z_{5}$ transition at $0.946 \mu \mathrm{m}$. $\mathrm{Nd}: \mathrm{YGG}$, that is $\mathrm{Nd}: \mathrm{Y}_{3} \mathrm{Ga}_{5} \mathrm{O}_{12}$, operates on the $R_{1}$ to $Z_{5}$ transition at $0.938 \mu \mathrm{m}$. By selecting the proper ratio of $\mathrm{Ga}$ and $\mathrm{Al}$, wavelengths between 0.946 and $0.938 \mu \mathrm{m}$ can be

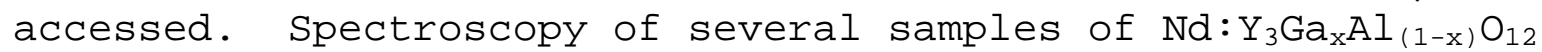
show nearly linear wavelength shift as $X$ is varied.

Laser operation of the $R_{1}$ to $Z_{5}$ transition can be efficient under either flash lamp or diode pumping. Before embarking on this project, the literature was consulted. The best reported $N d$ :YAG performance on the $R_{1}$ to $Z_{5}$ transition under flash lamp pumping was achieved at low temperatures, $248^{\circ} \mathrm{K}$. Performance is improved at reduced temperatures primarily because the thermal population density in the lower laser level is reduced. A threshold of $62 \mathrm{~J}$ and slope efficiency of 0.0014 was reported. By understanding the physics of this laser, an improved design was undertaken. With this design, a threshold of $12 \mathrm{~J}$ and a slope efficiency of 0.0096 were achieved at room temperature.

A laser diode pumped $\mathrm{Nd}$ laser operating on the $R_{1}$ to $Z_{5}$ transition achieved high efficiency. A lens duct concentrated the pump radiation from a laser diode array on a disk of laser material. Up to 3 disks of laser material could be deployed in a single laser resonator. Laser performance for this system appears in Figure 1 . Optical to optical slope efficiency of 0.288 was achieved with a single gain module in the resonator. Normal mode energy of $50 \mathrm{~mJ}$ was possible with a single gain module. 


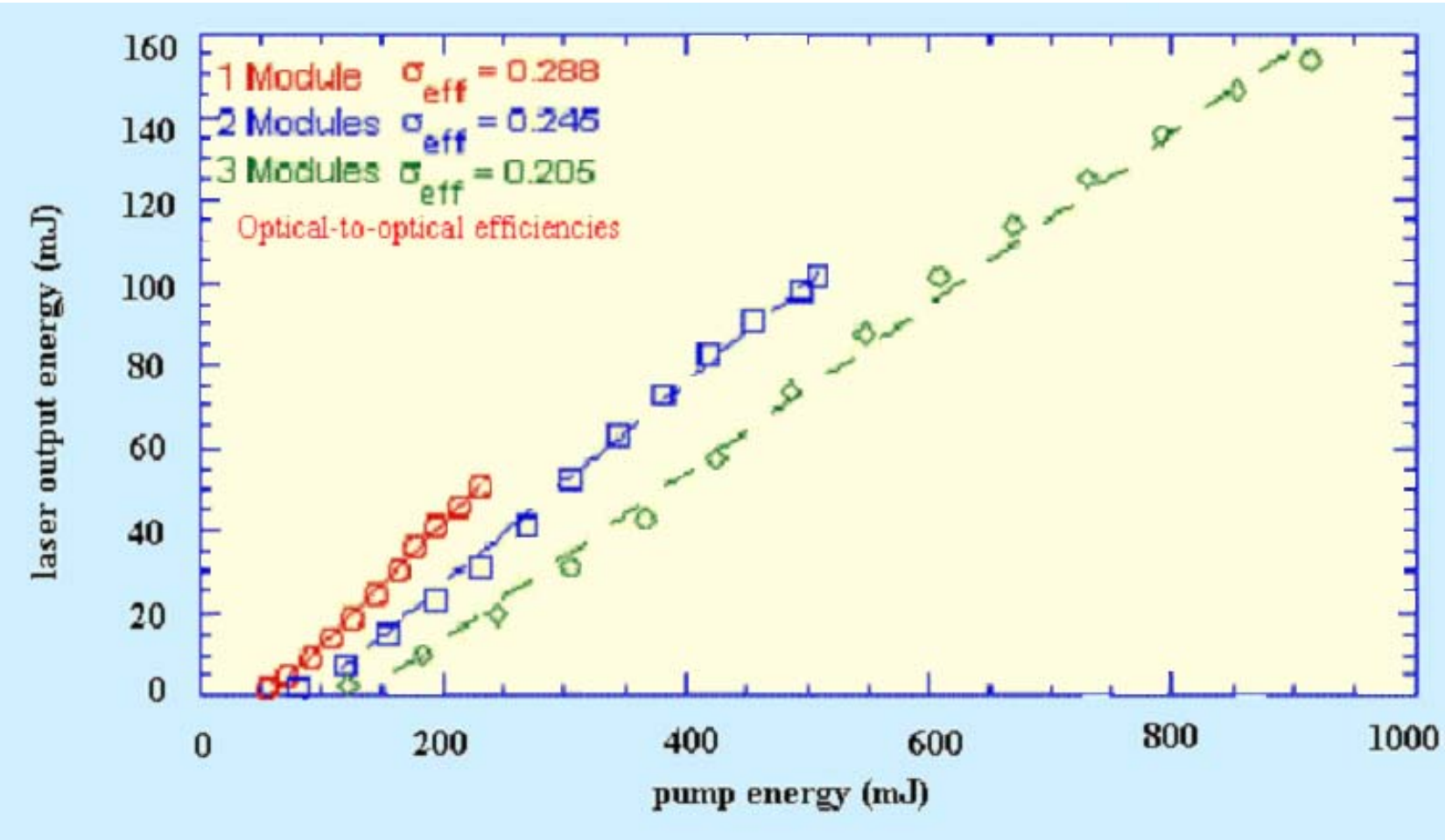

Figure 1. Normal mode performance of a Nd laser operating on the $R_{1}$ to $Z_{5}$ transition near $0.946 \mu \mathrm{m}$

\section{Nonlinear optics:}

Compositional tuning can be used to improve the efficiency of nonlinear optical devices. To be efficient, nonlinear optics interactions need to be phase matching. That is, conservation of energy and conservation of momentum need to be met. Thus,

$$
\begin{aligned}
& 1 / \lambda_{1}=1 / \lambda_{2}+1 / \lambda_{3}, \text { conservation of energy } \\
& n_{1} / \lambda_{1}=n_{2} / \lambda_{2}+n_{3} / \lambda_{3}, \text { conservation of momentum }
\end{aligned}
$$

where $\mathrm{n}_{i}$ is the index of refraction at wavelength $\lambda_{i}$. In normal dispersive materials, both equations can not be met because the index of refraction is a monotonically decreasing function of the wavelength. To overcome this, birefringent materials are usually used. In birefringent materials, there are 2 indices of refraction, polarization dependent, for a given direction of propagation. By varying the direction of propagation, phase matching can be satisfied. Although phase matching is achieved, some other birefringent effects may limit the efficiency.

A limiting effect arises when the interacting laser beams separate. Separation results in a birefringent crystal when the 
direction of energy propagation and the direction of phase propagation are not the same. Separation limits the effective interaction length and thus the efficiency of the interaction. Also, the first order variation of the refractive index with the angle of propagation results in a first order limitation on the range of incident angles that enjoy phase matching. This limit to the range of useful angles is referred to as the acceptance angle. If the range of incident angles exceeds the acceptance angle, the interaction will be less efficient. Both limitations can be overcome by using noncritcal phase matching.

If phase matching is achieved along an axis of the index ellipsoid, noncritical phase matching, both deleterious effects are mitigated. In this case the direction of energy propagation and the direction of phase propagation are the identical. This increases the useful length of the nonlinear crystal, therefore improving efficiency. Additionally, the first order dependence on the acceptance angle on the incident angle vanishes. The acceptance angle is still limited through a second order effect however it is an order of magnitude larger. Thus, a nonlinear crystal that achieves noncritical matching is sought for the nonlinear interaction. Compositional tuning can be used to address this problem as well.

Refractive indices of nonlinear crystals depend on their composition and are thus capable of being tuned. As an example, phase matching of KTA and RTA are shown in Figure 2. For these biaxial nonlinear crystals, the wavelength of the fundamental that achieves phase matching in the xy plane is displayed. By varying the azimuthal angle, KTA can be tuned to generate second harmonic between 1.09 and $1.15 \mu \mathrm{m}$ while RTA can be tuned between 1.15 and $1.25 \mu \mathrm{m}$. However, except for the end points, phase matching requires critical phase matching. On the other hand, noncritical phase matching can be sought using compositional tuning.

Noncritical phase matching should be possible between 1.15 and $1.25 \mu \mathrm{m}$ at $\odot^{\circ}$ azimuthal angle and between 1.09 and $1.15 \mu \mathrm{m}$ at $90^{\circ}$ azimuthal angle by varying the $\mathrm{Rb}$ to $\mathrm{K}$ ratio. As the $\mathrm{Rb}$ to $\mathrm{K}$ ratio is varied, the phase matching curves are expected to move between curves for stoichiometric RTA and KTA. In particular, phase matching can shift along vertical lines at $\odot^{\circ}$ and $90^{\circ}$ where noncritical phase matching exists. Once the nonlinear crystal is grown, the $\mathrm{Rb}$ to $\mathrm{K}$ ratio can not be changed. However, the compositionally tuned crystal will still have an angular tuning range that is similar to the related stoichiometric crystals. 


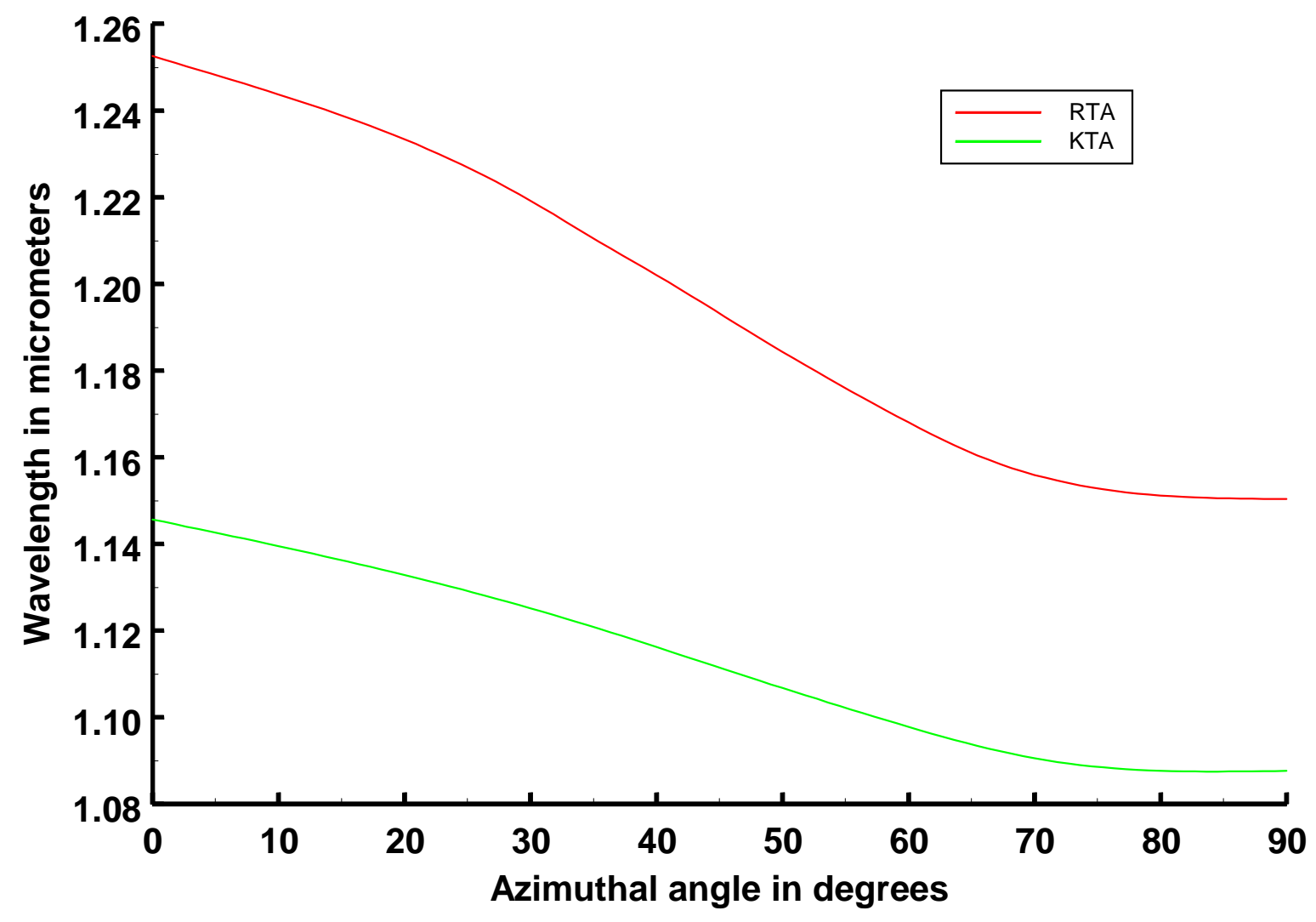

Figure 2. Angular and compositional tuning in RTA and KTA. Angular tuning adjusts the azimuthal angle and incurs birefringent effects. Compositional tuning proceeds along $\odot^{\circ}$ or $90^{\circ}$ vertical lines and minimizes deleterious effects.

\section{Summary:}

Compositional tuning can be used to tune lanthanide series laser material to a preselected wavelength. Quantum mechanical models have correctly predicted the laser wavelength and data needed to correctly laser performance. As an example, a laser material was invented to operate at a wavelength preselected by a remote sensing application, $0.9441 \mu \mathrm{m}$. Efficient performance was achieved despite the potential impediments of quasi 4 level operation and a competition from the strong $1.06 \mu \mathrm{m}$ transition.

Compositional tuning of nonlinear crystals can be used to achieve noncritical phase matching at any specified wavelength over a wavelength range. This wavelength range is associated with the particular crystals in a family of nonlinear crystals. By achieving noncritical phase matching, the deleterious effect of beam separation and small acceptance angle can be eliminated or reduced. Potential phase matching ranges were calculated for RTA and KTA. 